\title{
Heat-not-burn and electronic cigarettes: Truths and untruths about harm reduction
}

\author{
Francisco Paumgartten ${ }^{1 *}$ [6 \\ ${ }^{1} \mathrm{MD}$, PhD, National School of Public Health, Fundação Oswaldo Cruz, Rio de Janeiro, RJ, Brazil
}

*Correspondence:

The Editorial by the Brazilian Medical Association (AMB) ${ }^{1}$ warning physicians and the society against the use of electronic (EC) and heat-not-burn (HNB) cigarettes is very timely, given the powerful lobby for their approval in Brazil. ECs heat liquids containing nicotine, flavorings and propylene glycol, and/or glycerin into aerosols for inhalation. Since they do not mimic tobacco cigarettes' taste and flavor, they do not satisfy many smokers. Manufacturers' allegations that ECs are "safe" are questionable, and studies on the adversity of EC vapors to respiratory airways yielded conflicting results.

Recently, tobacco companies bet their chips on HNB cigarettes that they claim to be a "new wave of a harm reduction revolution." HNB tobacco is breaking into markets around the world, and a study suggested that consumption of this new type of nicotine delivery product is poised for explosive growth over the coming years. ${ }^{2}$ Unlike conventional cigarettes, HNB products heat the tobacco $\left(350^{\circ} \mathrm{C}\right)$ instead of burning it at higher temperatures (around $800^{\circ} \mathrm{C}$ ). While HNB devices aerosolize nicotine and deliver it efficiently to the bloodstream and brain, and give users a tobacco-flavored vapor, they do not produce carbon monoxide (CO) or generate carcinogens and pyrolysis-derived compounds that play a major role in the cigarette smoke toxicity. ${ }^{3}$ Thus, although it is plausible to think that replacing traditional cigarettes with HNB tobacco products would lower the risks of smoking-related diseases such as chronic obstructive pulmonary disease (COPD), lung cancer and some other conditions, this notion requires confirmation by epidemiological data. HNB tobacco, however, is not harmless. No matter whether nicotine is absorbed from tobacco vapor or from the smoke of cigarettes, it is one of the most addictive drugs. Nicotine addiction is a chronic and relapsing illness characterized by compulsive drug seeking and use despite user awareness about health risks and desire to quit. In other words, nicotine addiction per se is a major health hazard. Moreover, pharmacological and toxicological studies as well as some epidemiological investigations suggested that nicotine, in addition to $\mathrm{CO}$ and other smoke chemicals, contributes to cardiovascular events in smokers with underlying cardiovascular disease, and some authors believe that it accelerates formation of atheromatous lesions in arterial walls. ${ }^{4}$

Harm reduction policies and practices aim to minimize the harms associated with psychoactive drug consumption in people who are unable or unwilling to quit. That is, harm reduction focuses on the prevention of harms in people who continue to use drugs. In this line, as far as smoking is concerned, a distinction should be made between harm reduction at the individual patient and collective levels.

As pointed out by the AMB, "... there is no scientific evidence that the use of e-cigarettes is effective in reducing traditional cigarette smoking or in stopping smoking." Actually, a systematic review of randomized controlled trials (RCT) on the use of ECs for smoking cessation found two RCTs indicating that ECs (compared to placebo) aided smokers to quit smoking, and one trial showing no difference between ECs and nicotine patches. ${ }^{5}$ The authors, however, rated the confidence on the review conclusions as "low" (GRADE standards), owing to the small number of trials, low event rates and 
wide confidence intervals around the mean estimates. ${ }^{5}$ In other words, it remains unclear whether ECs are effective and, if so, whether they are more effective (and safe) than nicotine patches as smoking cessation treatments. The use of ECs to reduce harm in smokers at risk of, or with COPD, on the other hand, was unsupported by an observational study. ${ }^{6}$ A large prospective cohort study revealed that ECs were associated with worse pulmonary-related health outcomes, but not with cessation of smoking. ${ }^{6}$

Contrasting with ECs, which do not find full acceptance among tobacco smokers, HNB products provide smokers not only quickly absorbable nicotine but also typical tobacco flavor. Obviously, replacing HNB tobacco with traditional cigarettes does not reduce smokers' dependence on nicotine and so it is unlikely that they contribute for achieving abstinence. The use of HNB tobacco reduces exposure to toxic substances contained in tobacco smoke, which is expected to translate into lower risks of tobacco-associated chronic diseases. Epidemiology studies and/or RCTs, however, fail to substantiate the conjecture that long-term use of HNB tobacco is safer than smoking traditional cigarettes. At any rate, for patients addicted to nicotine who failed previous treatments with conventional smoking cessation medications, or are unwilling to quit smoking, HNB cigarettes may become a valid alternative for harm reduction.

A possible health benefit for a particular group of smokers, however, does not justify approval of HNB tobacco products for (unrestricted) sales. The tobacco industry's allegations that approval of HNB products for marketing would improve public health by reducing tobacco-associated deaths and diseases is at best an untested and self-serving hypothesis.
Were HNB products (and ECs) in fact less harmful than conventional cigarettes, they would still have the potential to cause addiction to nicotine (a major health hazard) and other adverse health effects. Moreover, marketed as supposedly healthier alternatives, HNB tobacco products would not only promote smoking appeal and initiation among young people, but also discourage older health-conscious smokers to seek treatment for their addiction to nicotine. Finally, if HNB tobacco products and traditional cigarettes coexist on the market, smokers can switch easily from one product to the other depending on a number of factors. In summary, it is foreseeable that unrestricted access to HNB cigarettes would make the quest for the first tobacco-free generation of Brazilians a distant or even unattainable public health goal. Approval of HNB products for use under medical supervision (prescription only) with advertising restrictions is a regulatory decision that would reconcile the needs of a group of nicotine-dependent patients and the collective goals in public health.

\section{References}

1. Comissão de Combate ao Tabagismo da Associação Médica Brasileira. AMB warns against the use of electronic delivery devices: electronic and heated cigarettes. Rev Assoc Med Bras. 2017; 63(10):825-6.

2. Caputi TL, Leas E, Dredze M, Cohen JE, Ayers JW. They're heating up: Internet search query trends reveal significant public interest in heat-notburn tobacco products. PLoS One. 2017; 12(10):e0185735.

3. Bekki K, Inaba Y, Uchiyama S, Kunugita N. Comparison of chemicals in mainstream smoke in heat-not-burn tobacco and combustion cigarettes. J UOEH. 2017; 39(3):201-7.

4. Benowitz NL, Burbank AD. Cardiovascular toxicity of nicotine: implications for electronic cigarette use. Trends Cardiovasc Med. 2016; 26(6):515-23.

5. Hartmann-Boyce J, McRobbie H, Bullen C, Begh R, Stead LF, Hajek P. Electronic cigarettes for smoking cessation. Cochrane Database Syst Rev. 2016; 9:CD010216.

6. Bowler RP, Hansel NN, Jacobson S, Graham Barr R, Make BJ, Han MK, et al. Electronic cigarette use in US adults at risk for or with COPD: analysis from two observational cohorts. J Gen Intern Med. 2017; 32(12):1315-22. 